\title{
Increase in developmental instability upon inbreeding in Daphnia
}

\author{
HONG-WEN DENG* \\ Department of Biology, University of Oregon, Eugene, OR 97403, U.S.A.
}

\begin{abstract}
Lerner's genetic homeostasis theory (1954) hypothesizes that developmental instability (DI) will be higher for individuals with lower genomic heterozygosity. Because the usual indices of DI may confound genetic effects with random developmental process, most previous tests of the hypothesis may have explanational difficulties. I shall present an alternative approach to test the genetic homeostasis hypothesis. In this approach, outcrossed and inbred genotypes, each having multiple clonal replicates, are assayed side-by-side in one controlled homogeneous and benign environment. Phenotypic variation among replicates for each genotype is used as a relative index for DI, not confounded by any possible genetic effect. The outcrossed genotypes differ from the inbred ones in the average genomic heterozygosity, thus avoiding the ambiguity of using heterozygosity at the allozyme level as an index for that at the genomic level. The approach may also provide data on the potential effects of genotypic value $(G)$ on DI. The results of experiments on two cyclically parthenogenetic Daphnia species (D. pulex and $D$. pulicaria) reveal that (i) for all characters analysed, DI increases upon inbreeding and (ii) there is no significant relationship between $G$ and DI. The increase in DI upon inbreeding is thus attributable only to a reduction in heterozygosity (per se or an enhanced expression of deleterious recessive alleles). Therefore, the present study is consistent with Lerner's genetic homeostasis hypothesis.
\end{abstract}

Keywords: cyclical parthenogenesis, Daphnia, developmental instability, genetic homeostasis, inbreeding.

\section{Introduction}

Genetic homeostasis is an old, but still very controversial, topic in evolution and genetics (Lerner, 1954; Wright, 1977; Falconer, 1981; Markow, 1993). It has many implications in biology, from an organism's development (Waddington, 1957), to individual adaptations to fluctuating environmental conditions (Lewontin, 1956, 1957) to populations' evolutionary response to selection (Deng \& Kibota, 1995) and, most interestingly, to a central question in evolutionary genetics, i.e. the preservation of genetic variability (Lerner, 1954; Lewontin, 1964; Gillespie \& Turelli, 1989; Zhivotovsky \& Feldman, 1992).

Lerner (1954) defined genetic homeostasis as a negative correlation between developmental instability (DI) and overall genomic heterozygosity. $\mathrm{He}$ believed that one reason for the high fitness of heterozygotes is their increased capacity to buffer

*Presentaddress: Human Genetics Center, SPH, University of Texas, Houston, TX 77225, U.S.A. E-mail: deng@hgc.sph.uth.tmc.edu environmental perturbations. This buffering may be envisaged from a biochemical-genetic point of view: when different allelic products have different optima along a range of environmental conditions, heterozygotes will have the most stable phenotype. Notable biochemical examples include sickle-cell haemoglobin in humans (Futuyma, 1986, Ch. 6) and alcohol dehydrogenase in maize (Schwartz \& Laughner, 1969).

The main prediction of the genetic homeostasis hypothesis is that DI will be higher for individuals with lower genomic heterozygosity (Lerner, 1954), which has been tested by many experiments with the following three main approaches.

The first has been to use two presumably completely inbred parental lines (P) and their first generation hybrid $\left(F_{1}\right)$. Each $F_{1}$ individual will be heterozygous at those loci fixed for different alleles in the two P lines. Within each of the three groups (two $P$ groups and $F_{1}$ ), all individuals are presumed to be genetically identical. The observed phenotypic variance of each group is thus taken to be the 
environmental variance $\left(V_{\mathrm{e}}\right)$, an index for DI for that group. Data from such experiments (e.g. Robertson \& Reeve, 1952; Dobzhansky \& Levene, 1955; Chai, 1957; Leamy \& Thorpe, 1984) reveal that $V_{\mathrm{e}}$ is almost always lower in the $\mathrm{F}_{1}$ than the average of the two $P$ lines (but see Lewontin, 1957 and Angus \& Schultz, 1983).

The second approach has been to compare the observed levels of phenotypic variation within natural populations with high vs. low heterozygosity for allozyme loci. Most data of this type provide support for the hypothesis (e.g. Eanes, 1978; Zouros et al., 1980; Livshits \& Kobyliansky, 1984), whereas some do not (e.g. Handford, 1980; King, 1984).

The third approach has been to study fluctuating asymmetry (FA) among groups of different genomic heterozygosity (reviewed in Palmer \& Strobeck, 1986; Mitton, 1993). Data from this approach generally reveal increased FA with increasing homozygosity, despite some exceptions (e.g. Zakharov, 1981; Livshits \& Smouse, 1993; Woolf, 1993).

Several sources of bias may lead to interpretational difficulties in all three approaches. In most studies with the first approach, the $\mathrm{P}$ lines were probably not completely homozygous. Thus, the observed phenotypic variance within each group must have contained a genetic component. With dominance and a reasonable range of gene frequencies in the two $P$ lines, the genetic variance in the $F_{1}$ can be considerably lower than the average of those in the two P lines (Lynch \& Walsh, 1996). In the second approach, in most analyses, the homozygous groups may contain individuals homozygous for different alleles. For a diallelic locus with alleles $A$ and $a$, in the extreme case, the homozygous group would comprise all $A A$ and $a a$ genotypes, whereas the heterozygous group would only consist of $A a$ genotypes. The homozygous groups are thus more genetically variable, which would probably result in a higher phenotypic variance. For a multilocus system (Chakraborty \& Ryman, 1983; Allendorf \& Leary, 1986), additive genetic variation may be sufficient to explain the phenotypic variation patterns among groups with different degrees of allozyme heterozygosity. Therefore, the observed difference of $V_{\mathrm{e}}$ (in the first approach) or phenotypic variance (in the second approach) could have been substantially influenced by genetic variance, in which case no special argument regarding the genetic homeostasis hypothesis is required. Furthermore, the second approach is based on the assumption that the level of heterozygosity revealed at a few allozyme loci strongly correlates with that of the whole genomic level, which itself is questionable from theory
(Mitton \& Pierce, 1980; Chakraborty; 1981, 1987) and from the few data available (M. Lynch and $\mathrm{K}$. Spitze, unpublished data).

The first approach was also criticized on different grounds by Waddington (1957) and Lande (1980), who pointed out the potential effects of genotypic value $(G)$ on DI. They postulated, from a purely theoretical point of view, that there is a positive quadratic relationship between DI and $G$, so that individuals with extreme $G$-values will have higher DI than those with intermediate $G$-values. Although no experiment has ever addressed this hypothesis explicitly, studies of phenotypic canalization (Waddington, 1957) and data from Soulé \& CuzinRoudy (1982) suggest such a possibility.

In the third approach to FA studies, the essential assumption that the two sides of a bilaterally symmetrical organism are under the control of the same set of genes has never been established by empirical evidence; neither has the assumption that the two sides always experience identical environments. Additionally, FA may not always be totally environmentally induced in all studies; it may have an underlying genetic component in some cases (Chippindale \& Palmer, 1993; Palmer et al., 1993). Furthermore, studies by Livshits \& Smouse (1993) do not support the view that FA is a good measure of DI of the whole organism. Although data from other lines of evidence are available, they either do not provide unequivocal results (e.g. Knowles \& Mitton, 1980; Ledig et al., 1983; Strauss, 1987) or are subject to the criticisms of Lande (1980) and/or Lynch \& Walsh (1996).

Here, I shall present experiments of an alternative approach, in which the relative index for DI is clearly separated from any possible genetic component of variance, and the potential effects of $G$ on DI can be checked. The questions to be addressed are as follows. (i) Do selfed individuals tend to have higher DI than outbred individuals? (ii) Is there any relationship (linear or quadratic) between $G$ and DI?

\section{Materials and methods}

The approach takes advantage of the special features of life cycles of cyclical parthenogens, which can clone their genotypes through ameiotic reproduction, and whose selfed offspring are relatively easily obtainable. Freshwater cladoceran species of Daphnia are such organisms. In their natural habitats, cyclically parthenogenetic Daphnia populations are generally outbred (Hebert, 1987). Individuals 
reproduce ameiotically (Hebert \& Crease, 1980) in nature and in the laboratory in favourable environmental conditions. Mitotic recombination is very rare or absent (Hebert, 1987). Genotypes can therefore be replicated faithfully (barring mutation) and represented multiple times within and among experiments. Selfed progeny can be obtained relatively easily in the laboratory by inducing sexual reproduction within parental clonal cultures and hatching sexually produced eggs (Innes, 1989; De Meester, 1993; Lynch \& Deng, 1994; Deng 1995, 1996a,b; Deng \& Lynch, 1996).

The Daphnia species used in the following experiments are $D$. pulex (or a close congener, M. Lynch, unpublished data) and $D$. pulicaria, collected, respectively, from Amazon Park in Eugene and Dorena Reservoir in Cottage Grove, both in Oregon, U.S.A. Females sampled from the field were isolated into individual beakers containing about $200 \mathrm{~mL}$ of water from the populations' source pond or reservoir, and they were fed with the green alga Scenedesmus. The species identity was determined by morphological (Brooks, 1957; Brandlova et al., 1972) and biochemical (Hebert et al., 1988, 1989; Lynch et al., 1989) criteria. Electrophoretic studies confirmed that both populations reproduce by cyclical parthenogenesis and are outbred (Lynch \& Deng, 1994; Deng \& Lynch, 1996). For each population, more than 300 clones isolated from their natural habitats were used as parental clones. The selfed clones were obtained by procedures described in Lynch \& Deng (1994). Briefly, over a period of a few months, the isolated females reproduced asexually, forming cohorts of genetically identical individuals. During this period, most clones also reproduced sexually, i.e. males were produced ameiotically, and some asexual females switched to sexual reproduction by producing sexual eggs meiotically. As males and females in each beaker are genetically identical, mating among them is genetically equivalent to selfing. The resultant sexually produced eggs are in a diapausing form (ephippia) and hatched by taking them through light/warm and dark/cold cycles (Lynch \& Deng, 1994; Deng \& Lynch, 1996). The hatched selfed individuals were then expanded clonally. The overall genomic heterozygosity of selfed clones is about half that of their parental clones (Crow \& Kimura, 1970).

To address the question relating genomic heterozygosity to DI, I performed inbreeding life table experiments with the Amazon and Dorena populations. Within each population, 30 random outcrossed clones and 30 random selfed clones (each from one of 30 random outcrossed parental clones different from those used in the experiment) were assayed simultaneously using the standard life table experimental design (Lynch, 1985; Lynch et al., 1989; Lynch \& Deng, 1994). Briefly, in the experiments, each clone was represented by three replicate lines, with each line started with an immature female from its clonal culture. Before measuring any life history traits, all the replicate lines were acclimatized to the standard experimental conditions (see below) for two asexual generations. Starting from the third generation, newborn individuals were measured daily (Amazon population) or every other day (Dorena population) from birth to the second (Amazon population) or the third (Dorena population) adult instar to obtain data for different life history traits, such as instar-specific body size, age at release of first clutch, growth rates.

The standard experimental conditions were as follows. All clonal lines were maintained in $100 \mathrm{~mL}$ of water (aged for at least 1 month and filtered before use) from the populations' source pond or reservoir, with approximately 300000 cells of the green alga Scenedesmus per mL. For the Amazon population, the experiment was conducted at $20^{\circ} \mathrm{C}$ (a typical daytime temperature during the growing season in the population's source habitat - a seasonal pond), $12 \mathrm{~h}: 12 \mathrm{~h}$ light-dark photoperiod (typical during this population's growing season) and the culture water was replenished every day. For the Dorena population, the experiment was conducted at $10^{\circ} \mathrm{C}$ (a typical temperature during the growing season in the population's source habitat - a permanent lake), $16 \mathrm{~h}: 8 \mathrm{~h}$ light-dark photoperiod (typical during this population's growing season) and the culture water was changed every other day (owing to the slow development of Daphnia at this temperature). The experimental conditions used are considered as optimal (high food, low density and constant temperature and photoperiod of the growing season) for Daphnia from the two distinct habitats (Lynch, 1989; Spitze et al., 1991).

In this approach, inbred and outbred clones are acclimatized and assayed side-by-side in one uniform environment, so that any change in genetic and developmental parameters between outcrossed parental and selfed progeny generations cannot be attributed to any temporal environmental change. Each clone is represented multiple times. For the phenotype of replicate lines of a genotype (a clone), the mean is an unbiased estimator for the genotypic value $(G)$, whereas the within-clone variance $\left(V_{\mathrm{e}}\right)$ is an ideal relative index for DI, not confounded by any genetic effect. Note that $V_{\mathrm{e}}$ is totally microenvironmental (including measurement error) and 
developmental in nature. Because the replicate lines of the outbred and inbred clones are distributed randomly in the highly controlled 'homogeneous' environment, the microenvironmental component of $V_{e}$ should not differ in the outbred and inbred groups, and thus will not bias $V_{\mathrm{e}}$ as an ideal indicator for comparing DI between the two groups, i.e. the difference in $V_{\mathrm{e}}$ in the inbred and outbred groups should result solely from developmental noise, nothing else. Thus, the higher the $V_{\mathrm{e}}$, the less stable is the developmental process.

To address the question of the relationship between $G$ and DI as indexed by $V_{\mathrm{e}}$, the following life table experiment was conducted on a large scale. Using the same experimental design, 181 clones from the outbred parental generation of the Dorena population were assayed. The controlled experimental conditions were the same as the Dorena inbreeding life table experiment. For each character of each clone, a pair of $G$ and $V_{\mathrm{e}}$ is available, providing data to test Waddington's (1957) and Lande's (1980) proposition.

\section{Data analyses and results}

For each character of each clone, an unbiased estimate of $V_{\mathrm{e}}$ was calculated by the standard formula for estimating sample variance when sample size $n$ is small:

$$
V_{\mathrm{e}}=\frac{\left.n \times \overline{\left(z^{2}\right.}-\bar{z}^{2}\right)}{n-1}
$$

where $\overline{z^{2}}$ is the mean of squares of the trait measurement and $\bar{z}^{2}$ is the square of the mean of the trait measurement.

The empirical distribution of $V_{\mathrm{e}}$ for all the traits analysed within the inbred and outbred groups showed significant departure from normality, as indicated by the Kolomogorov statistic in the UNIVARIATE procedure (SAS Institute, 1985, Ch. 54). Thus, a nonparametric statistical method, the Wilcoxon rank sum test, was used to test the homogeneity of $V_{\mathrm{e}}$ in the selfed and outbred groups. The only requirement of this test is that the samples from the two groups to be compared are random, which is fully met here. The null hypothesis to be tested is that the mean $V_{\mathrm{e}}$ for each character in the selfed group $\left(V_{\mathrm{e}}\right.$ (in)) is equal to that in the outbred group $\left(V_{e}(o u)\right)$. The analysis was conducted using the NPAR1WAY procedure (SAS Institute, 1990).

To measure the relative change in DI as reflected by $V_{\mathrm{e}}$, an index, RDI, is constructed. RDI is the difference in the mean $V_{\mathrm{e}}$ values between the inbred and the outbred groups divided by the latter, i.e.

$\mathrm{RDI}=\frac{V_{\mathrm{e}}(\mathrm{in})-V_{\mathrm{e}}(\mathrm{ou})}{V_{\mathrm{e}}(\mathrm{ou})}$

Upon inbreeding, if $\mathrm{RDI}=0$, then DI does not change. If $\mathrm{RDI}>0$, DI increases; otherwise, DI decreases.

The RDI of four life history traits and the associated probabilities from the Wilcoxon rank sum test for the null hypothesis $\left(V_{\mathrm{e}}\right.$ (in) $\left.=V_{\mathrm{e}}(\mathrm{ou})\right)$ for both populations are summarized in Table 1 . In the individual tests for each character at the 5 per cent level, for two out of four and three out of four traits in the Amazon and Dorena populations, respectively, $V_{\mathrm{e}}$ is significantly higher in the selfed than in the outbred group. RDI is as high as 3.60 in the Amazon population and 3.49 in the Dorena population. Even after applying the conservative sequential Bonferroni procedure (Holm, 1979; Rice, 1989) for the number of tests conducted within each species, the results remain significant for the length at maturity in the Amazon $D$. pulex population and for the length at birth for the first clutch newborns in the Dorena $D$. pulicaria population. The results are, therefore, strongly consistent with the prediction of Lerner's genetic homeostasis hypothesis.

Using data from 181 outbred clones from the Dorena population, for each of the four traits I performed quadratic regression analysis, using $V_{\mathrm{e}}$ as a dependent variable and $G$ as an independent variable. Significance of the coefficients was judged by whether the observed value of an estimate exceeded the two associated standard errors; none of them (linear or quadratic) did so (Table 2). Furthermore, all the $r^{2}$ values were less than 6 per cent, which indicated that in none of the four traits can more than 6 per cent of the variability of $V_{\mathrm{e}}$ be explained by that of $G$ in the quadratic regression model. Hence, there is no support for the proposition that the change in $V_{\mathrm{e}}$ may have been caused by a shift of $G$ upon inbreeding, although significant inbreeding depression has been detected for most traits analysed (Table 3; Deng, 1995).

\section{Discussion}

The approach presented here avoids the potential problems mentioned in the Introduction, providing an unambiguous index for comparing DI. Additionally, the potential effects of $G$ on DI can be checked. The results demonstrated higher DI in the selfed than in the outbred group in two Daphnia species from two distinct habitats. No significant 
Table 1 Summary of the RDIs and their associated Wilcoxon rank sum test probabilities $(P)$ for different life history traits in the Amazon Daphnia pulex population and the Dorena $D$. pulicaria population

\begin{tabular}{lll}
\hline & \multicolumn{2}{c}{ RDI $(P)$} \\
\cline { 2 - 3 } Character & $\begin{array}{l}\text { Amazon } \\
(D . \text { pulex })\end{array}$ & $\begin{array}{l}\text { Dorena } \\
(D . \text { pulicaria })\end{array}$ \\
\hline Length of maturity & $0.54^{*}(0.01)$ & $0.08(0.55)$ \\
Length at birth for the first clutch & $0.94(0.41)$ & $3.49^{*}(0.01)$ \\
First adult instar duration & $1.00(0.34)$ & $3.29^{*}(0.05)$ \\
Immature growth rate & $3.60^{*}(0.04)$ & $1.35^{*}(0.04)$ \\
\hline
\end{tabular}

${ }^{*} V_{\mathrm{e}}(\mathrm{in})-V_{\mathrm{e}}(\mathrm{ou})$ is significant at the $5 \%$ level in the individual test.

Table 2 Summary of the coefficients of the linear and quadratic terms, their associated standard errors and the $r^{2}$ in the quadratic regression model

\begin{tabular}{lrrr}
\hline Character & LC (SE) & QC (SE) & $r^{2}$ \\
\hline Length of maturity & $-0.047(0.173)$ & $0.012(0.054)$ & 0.007 \\
Length at birth for the first clutch & $-0.092(0.065)$ & $0.067(0.049)$ & 0.022 \\
First adult instar duration & $1.281(1.264)$ & $-0.115(0.100)$ & 0.052 \\
Immature growth rate & $-0.015(0.011)$ & $-1.438(1.306)$ & 0.025 \\
\hline
\end{tabular}

Data are from 181 Dorena Daphnia pulicaria clones.

LC and QC are, respectively, linear and quadratic term coefficients in the quadratic regression; SE, one standard error; $r^{2}$, proportion of the variation of DI explained by the variation of $G$ in the regression model.

Table 3 Means (M) and standard errors (SE) for the traits analysed in the selfed (in) and outcrossed (ou) groups

\begin{tabular}{lll}
\hline Character & M (in) (SE) & M (ou) (SE) \\
\hline Amazon Daphnia pulex & & \\
$\quad$ Length at maturity & $1.25(0.07)$ & $1.33(0.04)$ \\
$\quad$ Length at birth for the first clutch* & $0.53(0.01)$ & $0.56(0.01)$ \\
First adult instar duration* & $4.02(0.11)$ & $3.55(0.09)$ \\
$\quad$ Immature growth rate* & $0.101(0.006)$ & $0.119(0.004)$ \\
Dorena Daphnia pulicaria & & \\
$\quad$ Length at maturity* & $1.50(0.03)$ & $1.59(0.02)$ \\
$\quad$ Length at birth for the first clutch* & $0.62(0.01)$ & $0.65(0.01)$ \\
$\quad$ First adult instar duration & $6.60(0.20)$ & $6.17(0.17)$ \\
Immature growth rate* & $0.062(0.002)$ & $0.068(0.001)$ \\
\hline
\end{tabular}

${ }^{*}$ Traits for which genotypic means change significantly upon selfing.

relationship between $V_{\mathrm{e}}$ and $G$ was detected. Consequently, the results are consistent with the concept of Lerner's genetic homeostasis and lend support to theoretical models (Lewontin, 1964; Gillespie \& Turelli, 1989; Zhivotovsky \& Feldman, 1992) for invoking genetic homeostasis as a basic assumption.
The results here are concordant with Innes' (1989) work in D. obtusa, in which he found strong inbreeding depression for almost every life history trait measured, as well as an increased within-clone variation of selfed clones compared with outbred clones. The results are also consistent with, and may 
provide partial explanations for, the observation that, in natural Daphnia populations surveyed electrophoretically, there is generally an excess of heterozygosity (Hebert et al., 1982 and references within). Mitton \& Grant (1984), Allendorf \& Leary (1986) and Zouros \& Foltz (1987) reviewed previous empirical evidence for an association between heterozygosity at the allozyme level and fitnessrelated traits. They concluded that generally, despite some exceptions, there is a positive relationship. If heterozygosity revealed at the allozyme loci level does correlate closely with that of quantitative trait (fitness) loci, then genetic homeostasis may well contribute to the conservation of genetic variability.

Recently, Gavrilets \& Hastings (1994) proposed a quantitative genetics model for analysing DI. They reached essentially the same conclusion as Lerner (1954), that is that there is a negative association between DI and genomic heterozygosity. Their conclusion does not depend on the assumption about the effects of heterozygosity per se as Lerner's (1954) did. It is just an outcome of their assumption that DI has an additive genetic basis with a mean zero in the population. The present index of DI $\left(V_{\mathrm{e}}\right)$ is totally microenvironmentally or developmentally determined, as stated before; therefore, it is very unlikely that the observed results of the inflated DI $\left(V_{\mathrm{e}}\right)$ in the selfed group are caused by $V_{\mathrm{e}}$ having an additive genetic basis.

Using D. magna, Yampolsky \& Scheiner (1994) also tried to test the main prediction of Lerner's (1954) genomic homeostasis hypothesis: DI decreases with increasing genomic heterozygosity. They compared DI of clones grouped according to the allozyme heterozygosity at only three to five polymorphic loci. However, Mitton \& Pierce (1980) and Chakraborty $(1981,1987)$ have already shown from theory that heterozygosity at a few allozyme loci (even a dozen) does not provide an accurate indicator of an individual's genomic heterozygosity. Additionally, experimental data in Daphnia (M. Lynch and K. Spitze, unpublished observations) failed to reveal any relationship of heterozygosity at about 10 allozyme loci with that of the whole genome. However, by inbreeding, as in the present approach, the different level of genomic heterozygosity in the inbred and the outcrossed groups can be ascertained.

Although the present approach can test the main prediction of the genomic homeostasis hypothesis without any ambiguity, it may not (neither can all the previous experiments) distinguish whether an increase in DI (if any) results from a decrease in heterozygosity per se or from an enhanced expres- sion of deleterious recessive alleles upon inbreeding (Markow, 1993). To distinguish these two potential genetic causes of the change in $V_{\mathrm{e}}$, new experiments are needed. An experiment that may accomplish such a task may be as follows: obtain a large number of genotypes resulting from selfing an outcrossed clone for several generations. Then, compare $V_{\mathrm{e}}$ of the developmentally most stable offspring clone with that of the outcrossed source clone. If an increase in $V_{\mathrm{e}}$ upon inbreeding is only caused by decreasing heterozygosity per se, then it is impossible to obtain an offspring clone with about the same developmental stability as the outcrossed source clone, as genomic heterozygosity is decreased upon several generations of selfing. If an increase in $V_{\mathrm{e}}$ upon inbreeding results only from an enhanced expression of deleterious recessive alleles, then it is possible to obtain an offspring clone with about the same developmental stability as (or even higher than) the outcrossed source clone, as it is possible to obtain an offspring clone that has lower or about the same level of expression of deleterious recessive alleles as the outcrossed source clone. This kind of experiment is similar in spirit to those trying to distinguish dominance and overdominance as the genetic cause of inbreeding depression (Lynch \& Walsh, 1996). Experiments of this type are going to be time- and labour-consuming, but should be feasible in cyclical parthenogens, such as Daphnia, as a large number of genotypes can be obtained from selfing an outcrossed clone for several generations.

To apply the present approach to test the validity and generality of the main prediction of genomic homeostasis, cyclical parthenogenesis is not essential, as long as the organisms can be propagated clonally. Therefore, for plants that can be propagated vegetatively, the same approach can be employed.

\section{Acknowledgements}

I thank Z. Banks, Q. Tian, S.-K. Lee and S.-L. Yu for laboratory assistance, W. Bradshaw, D. Houle, R. Lande, M. Lynch, R. Palmer and J. Willis for discussions and helpful comments to improve the manuscript. The work was supported by National Science Foundation grant BSR 89-11038 to M. Lynch.

\section{References}

ALLENDORF, F. W. AND LEARY, R. F. 1986. Heterozygosity and fitness in natural populations of animals. In: Soulé, M. E. (ed.) Conservation Biology: the Science of Scarcity 
and Diversity, pp. 57-76. Sinauer, Sunderland, MA. ANGUS, R. A. AND SCHULTZ, R. J. 1983. Meristic variation in homozygous and heterozygous fish. Copeia, 1983, 287-299.

BRANDLOVA, J., BRANDL, Z. AND FERNANDO, C. H. 1972. The cladocera of Ontario with remarks on some species and distribution. Can. J. Zool., 50, 1373-1404.

BRooks, J. L. 1957. The Systematics of North American Daphnia. Academic Press, New Haven, CT.

CHAl, C. K. 1957. Developmental homeostasis of body growth in mice. Am. Nat., 85, 49-55.

CHAKRABORTY, R. 1981. The distribution of the number of heterozygous loci in an individual in natural populations. Genetics, 98, 461-466.

CHAKRABORTY, R. 1987. Biochemical heterozygosity and phenotypic variability of polygenic traits. Heredity, 59, 19-28.

CHAKRABORTY, R. AND RYMAN, N. 1983. Relationship of mean and variance of genotypic values with heterozygosity per individual in a natural population. Genetics, 103, 149- 152.

Chippindale, A. K. AND Palmer, A. R. 1993. Persistence of subtle departure from symmetry over multiple molts in individual brachyuran crabs: relevance to developmental stability. Genetica, 89, 185-199.

CROW, J. AND Kimura, M. 1970. An Introduction to Population Genetics Theory. Harper \& Row, New York.

DE MEESTER, L. 1993. Inbreeding and outbreeding depression in Daphnia. Oecologia, 96, 80- 84.

DENG, H.-w. 1995. Sexual Reproduction in Daphnia: its Control and Genetic Consequences. PhD thesis. University of Oregon.

DENG, H.-w. 1996a. Environmental and genetic control of sexual reproduction in Daphnia. Heredity, 76, 449-458.

DENG, H.-w. 1996b. Photoperiodic response of sexual reproduction in Daphnia pulex group is reversed in two distinct habitats. Limnol. Oceanog. (in press) .

DENG, H.-W. AND KIBOTA, T. 1995. The importance of the environmental variance-covariance structure in predicting evolutionary response. Evolution, 49, 572-574.

DENG, H.-w. AND LYNCH, M. 1996. Change of genetic architecture in response to sex. Genetics, 143, 203-212.

DOBZHANSKY, T. AND LEVENE, H. 1955. Developmental homeostasis in natural populations of Drosophila pseudoobscura. Genetics, 40, 797-808.

EANES, w. F. 1978. Morphological variance and enzyme heterozygosity in the monarch butterfly. Nature, 276, 263-264.

FALCONER, D. s. 1981. Introduction to Quantitative Genetics, 2nd edn. Longman, New York.

FUTUYMA, D. J. 1986. Evolutionary Biology. Sinauer, Sunderland, MA.

GAVRILETS, S. AND HASTINGS, A. 1994. A quantitative genetic model for selection on developmental noise. Evolution, 48, 1478-1486.

GILLESPIE, J. H. AND TURELLI, M. 1989. Genotype-environmental interactions and the maintenance of polygenic variation. Genetics, 121, 129-138.
HANDFORD, P. 1980. Heterozygosity at enzyme loci and morphological variation. Nature, 286, 261-262.

HEBERT, P. D. N. 1987. Genetics of Daphnia. Memorie Instituto Italiano Di Idrobiologia, 45, 439-460.

HEBERT, P. D. N. AND CREASE, T. J. 1980. Clonal coexistence in Daphnia pulex (Leydig): another planktonic paradox. Science, 207, 1363-1365.

HEBERT, P. D. N., FERRAR1, D. C. AND CREASE, T. J. 1982. Heterosis in Daphnia: a reassessment. Am. Nat., 119, 427-434.

HEBERT, P. D. N., WARD, R. D. AND WEIDER, L. J. 1988. Clonal-diversity patterns and breeding-system variation in Daphnia pulex, an asexual-sexual complex. Evolution, 42, 147-159.

HEBERT, P. D. N., BEATON, M. J. AND SCHWARTZ, s. s. 1989. Polyphyletic origin of asexuality in Daphnia pulex. I. Breeding system variation and levels of clonal diversity. Evolution, 43, 1004-1015.

HOLM, s. 1979. A simple sequentially rejective multiple test procedure. Scand. J. Stat., 6, 65-70.

INNES, D. J. 1989. Genetics of Daphnia obtusa: genetic load and linkage analysis in a cyclical parthenogen. J. Hered., 80, 6-10.

KING, D. P, F. 1984. Enzyme heterozygosity associated with anatomical character variance and growth in the herring (Clupea harengus L.). Heredity, 54, 289-296.

KNOWLES, P. AND MITTON, J. B. 1980. Genetic heterozygosity and radial growth variability in Pinus contorta. Silvae Genet., 29, 114-118.

LANDE, R. 1980. Genetic variation and phenotypic evolution during allopatric speciation. Am. Nat., 116, 463-479.

LEAMY, L. AND THORPE, R. s. 1984. Morphometric studies in inbred and hybrid house mice. Heterosis, homeostasis and heritability of size and shape. Biol. J. Linn. Soc., 22, 233-241.

LEDIG, F. T., GURIES R. P. AND BONEFELD, B. A. 1983. The relation of growth to heterozygosity in pitch pine. Evolution, 37, 1227-1238.

Lerner, 1. M. 1954. Genetic Homeostasis. Oliver \& Boyd, Edinburgh.

LEWONTIN, R. C. 1956. Studies on homeostasis and heterozygosity. I. General consideration. Abdominal bristle number in second chromosome homozygotes of Drosophila melanogaster. Am. Nat., 90, 237-255.

LEWONTIN, R. C. 1957. The adaptation of populations to varying environments. Cold Spring Harb. Symp. Quant. Biol., 22, 359-408.

LEWONTIN, R. C. 1964. The interaction of selection and linkage. II. Optimum models. Genetics, 50, 757-782.

LIVSHITS, G. AND KOBYLIANSKY, E. 1984. Comparative analysis of morphological traits in biochemically homozygous and heterozygous individuals from a single population. J. Hum. Evol., 13, 161-171.

LIVSHITS, G. AND SMOUSE, P. E. 1993. Relationship between fluctuating asymmetry, morphological modality and heterozygosity in an elderly Israeli population. Genetica $, 89,155-161$. 
LYNCH, M. 1985. Spontaneous mutations for life-history characters in an obligate parthenogen. Evolution, 39, 804-818.

LYNCH, M. 1989. The life history consequences of resource depression in Daphnia pulex. Ecology, 70, 246-256.

LYNCH, M. AND DENG, H-W. 1994. Genetic slippage in response to sex. Am. Nat., 144, 242-261.

LYNCH, M., AND WALSh, B. 1996. Principles of Evolutionary Quantitative Genetics. Sinauer, Sunderland, MA (in press).

LYNCH, M., SPITZE, K. AND CREASE, T. 1989. The distribution of life-history variation in the Daphnia pulex complex. Evolution, 43, 1724-1736.

Markow, т. A. 1993. Developmental Instability: its Origins and Evolutionary Implications. Kluwer Academic Publishers, Boston, MA.

MITTON, J. B. 1993. Enzyme heterozygosity, metabolism, and developmental stability. Genetica, 89, 47-65.

MitTon, J. B. AND GRANT, M. c. 1984. Associations among protein heterozygosity, growth rate, and developmental homeostasis. Ann. Rev. Ecol. Syst., 15, 479-499.

MITTON, J. B. AND PIERCE, B. A. 1980. The distribution of individual heterozygosity in natural populations. Genetics, 95, 1043-1054.

PAlMER, A. R. AND STROBECK, s. 1986. Fluctuating asymmetry, measurement, analysis, patterns. Ann. Rev. Ecol. Syst., 17, 391-421.

PALMER, A. R., STROBECK, C. AND CHIPPINDALE, A. K. 1993. Bilateral variation and the evolutionary origin of macroscopic asymmetries. Genetica, 89, 209-218.

RICE, W. R. 1989. Analyzing tables of statistical tests. Evolution, 43, 223-225.

ROBERTSON, F. W. AND REEVE, E. C. R. 1952. Heterozygosity and environmental variation and heterosis. Nature, $170,286$.

SAS USER's GUIDE 1985. BASICS, Version 5. SAS Institute
Inc., Cary, NC.

SAS/STAT USER's guide. 1990. Volume 1, Version 6, 4th edn. SAS Institute, Cary, NC.

SCHWARTZ, D. AND LAUGHNER, w. J. 1969. A molecular basis for heterosis. Science, 166, 626-627.

SOULÉ, M. E. AND CUZIN-ROUDY, J. 1982. Allometric variation. 2. Developmental instability of extreme phenotypes. Am. Nat., 120, 765-786.

SPITZE, K., BURNSON, J. AND LYNCH, M. 1991. The covariance structure of life-history characters in Daphnia pulex. Evolution, 45, 1081-1090.

STRAuss, s. H. 1987. Heterozygosity and developmental stability under inbreeding and crossbreeding in Pinus attenuata. Evolution, 41, 331-339.

Waddington, c. H. 1957. The Strategy of the Genes. Allen \& Unwin, New York.

woolf, C. M. 1993. Does homozygosity contribute to the asymmetry of common white leg markings in the Arabian horse? Genetica, 89, 25-33.

WRIGHT, s. 1977. Evolution and the Genetics of Populations, vol. 3, Experimental Results and Evolutionary Deductions. The University of Chicago Press, Chicago.

YAMPOLSKY, L. Y. AND SCHEINER, s. 1994. Developmental noise, phenotypic plasticity, and allozyme heterozygosity in Daphnia. Evolution, 48, 1715-1722.

zAKHAROV, v. M. 1981. Fluctuating asymmetry as an index of developmental homeostasis. Genetica, 13, 241-256.

zHIVOTOVSKY, L. A. AND FELDMAN, M. w. 1992. On models of quantitative genetic variability: a stabilizing selection-balance model. Genetics, 130, 947-955.

zouros, E. AND FOLTZ, D. w. 1987. The use of allelic isozyme variation for the study of heterosis. Isozymes, 13, 1-59.

zOUROS, E., SINGH, M. AND MILES, H. 1980. Growth rate in oysters: an overdominant phenotype and its possible explanation. Evolution, 34, 856-867. 